\title{
Globular clusters in low mass galaxies
}

\author{
Margarita E. Sharina $^{1}$, T. H. Puzia ${ }^{2}$, V. L. Afanasiev ${ }^{1}$, \\ D. I. Makarov ${ }^{1}$, A. E. Dolphin ${ }^{3}$ and G. M. Karataeva ${ }^{4}$ \\ ${ }^{1}$ Special Astrophysical Observatory, Russian Academy of Sciences, \\ N. Arkhyz, KChR, 369167, Russia \\ emails: sme@sao.ru,vafan@sao.ru,dim@sao.ru \\ ${ }^{2}$ Space Telescope Science Institute, 3700 San Martin Drive, Baltimore, MD21218, USA \\ email: tpuzia@stsci.edu \\ ${ }^{3}$ Steward Observatory, University of Arizona, \\ 933 North Cherry Avenue, Tucson, AZ 85721, USA \\ email: adolphin@as.arizona.edu \\ ${ }^{4}$ Astronomical Institute, St. Petersburg State University, \\ Universitetsky pr., 28 Petrodvoretz, St. Petersburg 198504, Russia \\ email: narka@astro.spbu.ru
}

\begin{abstract}
We study properties of globular cluster candidates (GCCs) and the diffuse stellar populations in nearby low surface brightness dwarf galaxies, using HST WFPC2 photometry in the $\mathrm{V}$ and I bands. Our sample consists of 18 dwarf spheroidal (dSph), 36 dwarf irregular (dIrr), and 3 transition-type galaxies, with projected linear diameters less than $3.5 \mathrm{kpc}$ and mean blue surface brightness $>23 \mathrm{mag} / \operatorname{arcsec}^{2}$ situated at the distance 2-6 Mpc in the field and in nearby groups. Our sample dwarf spheroidal galaxies were not detected in HI and are located at the distances of up to $\sim 1 \mathrm{Mpc}$ away from a nearby bright galaxy. Transition-type galaxies, which are distributed like dSph galaxies, form a very rare class of galaxies. dIrr galaxies show a weaker concentration to the nearest massive neighbors than $\mathrm{dSph}$ and transition-type galaxies. At a given surface brightness and luminosity, they exhibit lower mean metallicities than dSphs. In contrast to dIrr galaxies, the majority of dSph galaxies at a similar mean surface brightness contains GCCs. The percentage of GCCs located near the centers of dSph galaxies is much higher than that for dIrr galaxies. The composite population of GCCs in dSphs is spatially more concentrated than in dIrr galaxies. The color distributions of GCCs in dSph and dIrr galaxies show major differences. While the latter shows obvious bimodality with the peaks near $(V-I)_{0} \sim 0.5$ and $\sim 1.0 \mathrm{mag}$, the GCC color distribution in dSph galaxies shows only one peak with a mean color $(V-I)_{0} \sim 1.0$ mag. There is a tendency of increasing half-light radii with increasing projected galactocentric distances for a large number of GCCs in dSph galaxies, which is also observed in the Galactic globular cluster system. We embarked on a spectroscopic survey of GCCs in low-mass galaxies to obtain their chemical compositions and relative ages.
\end{abstract}

Keywords. photometry, dwarf galaxies, star clusters, fundamental parameters

\section{Properties of our sample galaxies}

We select low surface brightness dwarf galaxies with accurately measured distances situated in the field and in the nearby groups: M81, Centaurus A, Sculptor, Canes Venatici I cloud. More than $50 \% \mathrm{dSph}$ are located within the projected radius of $250 \mathrm{kpc}$ from the nearest massive galaxy (see Fig. 1), which is well reproduced by CDM models (e.g. Kravtsov et al. 2004).

The galaxies were surveyed with the HST WFPC2 (GO-8192, GO-8601) with 600-s. expositions taken in the F606W and F814W filters. Photometry and artificial star tests were performed using DOLPHOT program (Dolphin 2000). We estimate the metallicity 


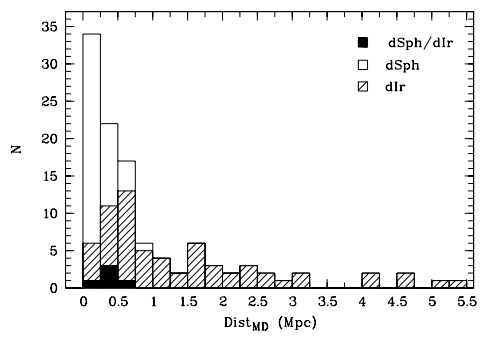

Figure 1. Distribution of nearby galaxies (our sample + LG) according to their projected distances from the nearest massive galaxy, Dist $t_{M D}(\mathrm{Mpc})$, from Karachentsev et al. (2004)
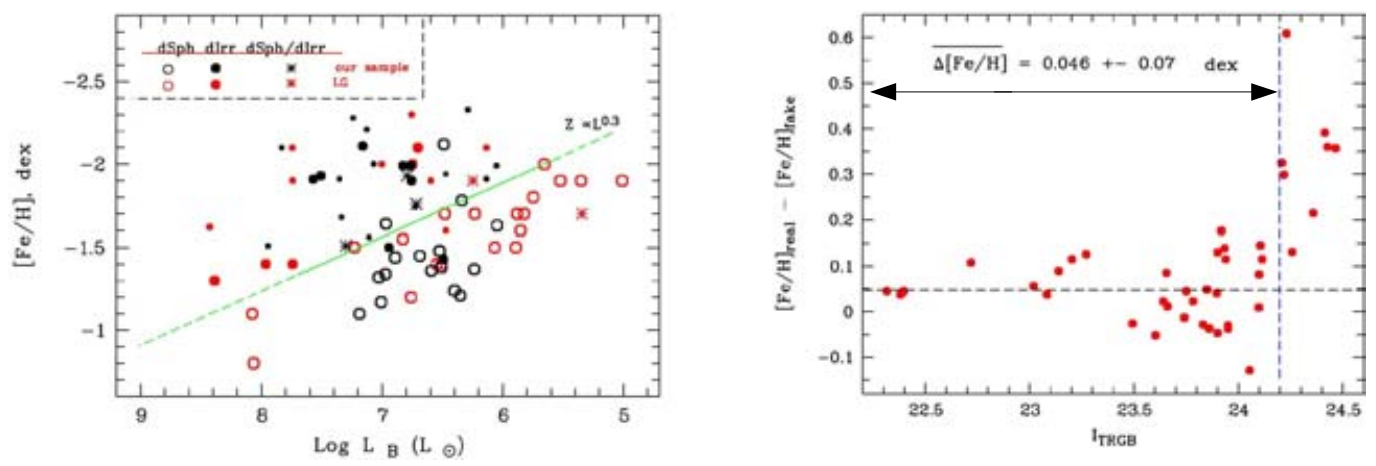

Figure 2. (a) B-band luminosity versus metallicity of red giant branch stars in our sample galaxies. Isolated dIrr galaxies (with tidal index, $T I<0$, according to Karachentsev et al. (2004)) are marked by small solid dots. The data for the LG galaxies were taken from Grebel et al. (2003). (b) Comparison of metallicity estimated from real and artificial RGB stars in dependence of apparent I magnitude of TRGB in our sample galaxies.

of RGB stars from the mean color of the red giant branch measured at an absolute magnitude $M_{I}=-3.5^{m}$ (Lee et al. 1993 ): $[\mathrm{Fe} / \mathrm{H}]=12.64+12.6(V-I)_{-3.5}-3.3(V-I)_{-3.5}^{2}$. We corrected our measurements using artificial star tests (see Fig. 2b). Fig. 2a shows that dSph galaxies follow a galactic metallicity-luminosity relation $\left(Z \sim L^{0.3}\right)$ fairly well, but dIrr galaxies have higher metallicity for their luminosity as compared to dSphs.

\section{Properties of globular cluster candidates}

Globular cluster candidates were selected and photometry was performed using the FIND task of the DAOPHOT-II Stetson(1987) package. To convert instrumental magnitudes F606W and F814W into the standard Johnson-Cousins system we used the surface photometry recipes and equations presented by Holtzman et al. (1995). The magnitudes of GCCs were corrected for Galactic extinction using reddening maps from Schlegel et al. (1998). We select GCCs with half-light radii of $2<\mathrm{FWHM}<9$ pix (corresponds to $3<r_{\mathrm{h}}<20 \mathrm{pc}$, which is typical for Galactic GCs), absolute magnitudes $M_{V}<-5.0^{m}$ and color $0.3<(V-I)_{0}<1.5$. We fit surface brightness profiles of our GCCs with the King law (King 1962). About $10 \%$ of the sample was removed from our GCCs list due to uncertain structural parameters. The final list contains 105 GCCs. In general, $50 \%$ of all surveyed galaxies contain globular cluster candidates, irrespective of morphological type.

Figure 3a shows a color-magnitude diagram for all GCCs. The red peak GCC subpopulation in dIrrs and most GCCs in dSph galaxies cover the same $(V-I)_{0}$ color range 


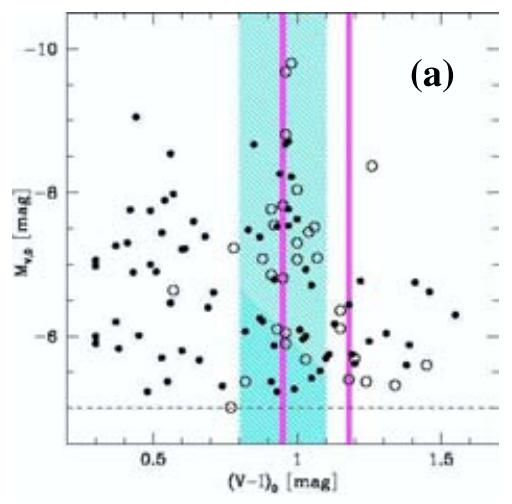

(b)

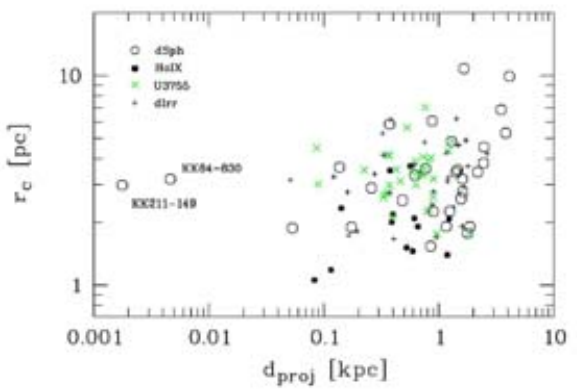

Figure 3. (a) Color-magnitude diagram of globular cluster candidates in dSph (open circles) and dIrr galaxies (solid dots). The dashed line indicates the limit of our photometry The hatched region shows the color range for Galactic and M31 globular clusters (Puzia et al. 2004). The two vertical lines indicate colors of blue and red sub-populations in massive early-type galaxies. (b) Core radius of GCCs versus their projected galactocentric distance. The data for the two richest GCC systems, UGC 3755 and Holmberg IX, are marked by different symbols.

as the ancient Galactic and M31 globular clusters and are similar to blue globular clusters in early-type galaxies, which implies similar ages and metallicities. For GCCs in dSphs the LF shows a turnover at around $M_{V} \approx-7.4^{m}$. We find indications for an excess population of faint GCCs with $M_{V}>-6.5$ in both dSph and dIrr, reminiscent of excess populations of faint GCs in the LG spiral galaxies. Figure $3 \mathrm{~b}$ shows a trend of increasing half-light and core radius as a function of increasing galactocentric distance. We find that most GCCs have structural parameters similar to extended outer halo globular clusters in the Milky Way and M31, as well as the recently discovered population of "faint fuzzy" clusters in nearby lenticular galaxies (Brodie \& Larsen 2002).

We find that the surface density profiles of globular cluster systems in dIrr galaxies are "flatter" $(x \approx 1.1)$ and $(x \approx 1.85)$ than those in dSph galaxies $(x \approx 2)$ (see Fig. 4$)$.

It should be mentioned, that in contrast to dIrr galaxies, $\sim 60 \%$ of dSphs with GCCs have cluster candidates located near their galaxy center. These GCCs are bright and compact $\left(-7<M_{V, 0}<-10, r_{c} \approx 2-3 \mathrm{pc}\right)$, similar to those found at the center of nucleated Virgo dE galaxies (Lotz et al. 2004).
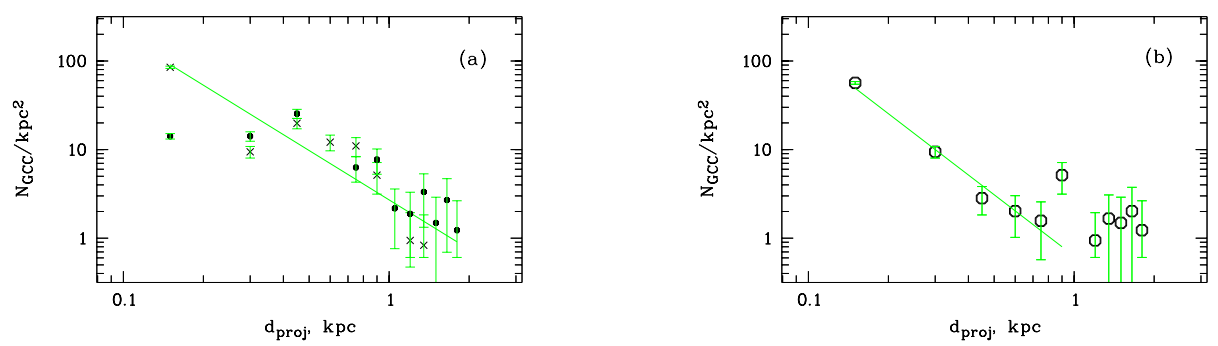

Figure 4. Radial distribution of GCCs in dIrr galaxies (panel a) and dSph galaxies (panel b). The sample of GCCs in dIrrs is divided into blue GCCs with $(V-I)_{0}<0.75$ (dots) and red GCCs $(V-I)_{0}>0.75$ (crosses). Plotted here is the logarithm of the surface density of GCCs per square kpc (evaluated in 0.15 -kpc bins) vs. the logarithmic projected distance from the galaxy center, in kpc. 


\section{Conclusions}

Our findings do not contradict a hypothesis that dSph galaxies turned their gas reservoirs early and more efficiently into stars than dIrrs of similar luminosity. dSph galaxies have higher metallicity of red giant stars for their luminosity as compared to dIrr galaxies. The detection rate of GCCs in dSph galaxies is $\sim 2$ times higher than in dIrr galaxies in the same surface brightness range $\mu_{B} \geqslant 24.4 \mathrm{mag} / \mathrm{arcsec}^{2}$. GCC systems of dSph galaxies are more spatially concentrated than in dIrr galaxies. The fraction of GCCs located near the center of dSph galaxies is 2 times higher than that for dIrrs. However, it is worth to note that dSph, dIrr and transition type galaxies host GCCs with $(V-I)_{0} \approx 1.0^{m}$, which might indicate that at least early globular cluster formation took place irrespective of the host galaxy's morphological type.

If accretion of globular clusters plays an important role in the assembly of outer globular cluster systems in massive galaxies, one should expect similarities in magnitudes and structural parameters between clusters in our sample dwarf galaxies and the outskirts of the Local Group spirals (Harris 1996). For GCCs in dSphs the luminosity function shows a turnover at around $M_{V}=-7.4$. Roughly half of our globular cluster candidates have structural parameters similar to extended outer halo globular clusters in the Local group spiral galaxies.

An ongoing spectroscopic survey of globular clusters in low-mass nearby galaxies will help us to reject background galaxies from our list and study the chemical composition and relative ages of globular clusters in low surface brightness dwarf galaxies.

\section{Acknowledgements}

M. E. S. would like to acknowledge IAU grant, RFBR travel grant 05-02-26517 and the conference organizers for a stimulating meeting.

\section{References}

Dolphin, A.E. 2000, PASP 112, 1383

Brodie, J.P. \& Larsen, S.S. 2002, AJ 124, 1410

Grebel, E.K., Gallagher, J.S. \& Harbeck, D. 2003, AJ 125, 1926

Harris, W.E. 1996, AJ 112, 1487

Holtzman, J.A., Burrows, C.J., Casertano, et al. 1995, PASP 107, 1065

Karachentsev, I.D., Karachentseva, V.E., Huchtmeier, W.K. \& Makarov, D.I. 2004, AJ 127, 2031

King, I. 1962, AJ 67, 471

Kravtsov,A.V., Gnedin, O.Y. \& Klypin, A.A. 2004, ApJ 609, 482

Lee, M.G., Freedman, W.L. \& Madore, B.F. 1993, ApJ 417, 553

Lotz, J.M., Miller, B.W. \& Ferguson, H.C. 2004, ApJ 613, 262

Puzia, T.H. et al. 2004, A\&A 415, 123

Schlegel, D.J., Finkbeiner, D.P \& Davis M. 1998, ApJ 500, 525

Stetson, P.B. 1987 PASP 99, 191

\section{Discussion}

MiLleR: What are the specific frequencies for globular cluster candidates in dIrrs?

ShARINA: A mean value of the specific frequency for GCCs in our sample dIrrs is $\sim 15$. 